\title{
Current Management of Undescended Testes
}

\author{
David Kurz, $M D^{1}$ \\ Gregory Tasian, MD, MSC, MSCE ${ }^{2, *}$
}

\author{
Address \\ ${ }^{1}$ University of Pennsylvania Perelman School of Medicine, Philadelphia, PA, USA \\ ${ }^{*}, 2$ Division of Urology and Center for Pediatric Clinical Effectiveness, The Children's \\ Hospital of Philadelphia, University of Pennsylvania Perelman School of Medicine, \\ Philadelphia, PA, USA \\ Email: tasiang@email.chop.edu
}

Published online: 25 January 2016

C Springer International Publishing AG 2016

\section{This article is part of the Topical Collection on Pediatric Urology}

Keywords Cryptorchidism • Undescended testicle (UDT) • Orchiopexy • Infertility · Testicular malignancy

\section{Opinion statement}

Undescended testes (UDTs) are a relatively common finding in newborn males, especially in those born prematurely. Upon discovering a non-intrascrotal testis, it is important to determine whether the testis is palpable or non-palpable and whether the finding is unilateral or bilateral. Imaging should not be used in this workup, as no current modality has been shown to be adequately sensitive or specific to aid in management decisions. Patients with UDTs diagnosed after 6 months of age should be referred to a specialist for correction so that surgery may be performed within 1 year thereafter. This allows testes to descend spontaneously if they are to do so while facilitating early intervention to decrease the risk of subfertility and testicular malignancy for those patients in whom spontaneous descent does not occur. The surgical approach is often dependent on the location of the testis on physical exam. Most orchiopexies for palpable testes are performed through an inguinal incision, although a scrotal approach can be safely utilized depending on the testis position. Diagnostic laparoscopy is most often used for non-palpable testes, as it not only allows for the identification of an atrophic or absent testicle, but it also provides an opportunity to perform an orchiopexy simultaneously should a viable testis be found. Hormonal therapy is not recommended for treatment of UDTs due to its low success rate, the incidence of secondary re-ascent, and the possible detrimental effects on spermatogenesis. Finally, patients with bilateral non-palpable UDTs require a more extensive preliminary evaluation to rule out congenital adrenal hyperplasia (CAH) and disorders of sexual development (DSD). This involves serum electrolytes, karyotype analysis, and hormonal testing including a serum müllerian inhibiting substance (MIS), in order to determine if testicular tissue is present and functional. 


\section{Introduction}

The undescended testicle (UDT), also referred to as cryptorchidism, is defined as the failure of the testis to descend into the scrotal position. The testicle may be present and extrascrotal, or it may be absent altogether. Cryptorchidism can either be congenital or acquired. Congenital cases involve an extrascrotal testicle from birth, while acquired UDTs are initially located in the scrotum at birth, but later become extrascrotal.

Several other terms help to further classify an acquired UDT. An ascending testicle is one where the testicle spontaneously moves extrascrotally from an intrascrotal position after birth. It is considered entrapped if the testicle migrates from the scrotal position after previous inguinal surgery. A retractile testis is located or moves outside of the scrotum (commonly resulting from a pronounced cremasteric reflex), but is able to be manually brought down into the scrotum without tension during a physical exam and remain in the dependent scrotum for a period of time after release. An atrophic testicle is one that is significantly smaller than the age-appropriate size either due to developmental failure, long-standing cryptorchidism, or previous surgery. A vanishing testis is one that was previously present and functional, but some insult during development (often a prenatal vascular event) caused it to regress. An absent testicle is defined at the time of surgery by identification of blind-ending testicular vessels.

\section{Background}

Cryptorchidism is one of the more common birth anomalies. Estimates range from 1 to $3 \%$ of full-term newborn males being affected by one or both testes failing to descend [1]. During fetal development, testicular descent occurs in two phases: a transabdominal phase in the first trimester and an inguinoscrotal phase between 25 and 30 weeks [2]. Spontaneous testicular descent occurs most commonly prior to 6 months and less commonly between 6 and 12 months after correcting for gestational age $[3,4]$. Several risks factors are known to play a role in cryptorchidism. It is more common in prematurity, as $15-30 \%$ of premature males have cryptorchidism in the first few months after birth. Family history has been associated with a higher risk, with certain genetic mutations likely contributing $[3,5]$. Other factors that may be associated with cryptorchidism include low birth weight for gestational age, as well as maternal smoking, analgesic use, and estrogen (DES) exposure during pregnancy [6-8].

\section{Diagnosis}

A physical exam should be performed to determine the position and morphology of the testicles. A UDT can be located in several locations along the path of descent (abdomen, inguinal canal, superficial inguinal pouch, or upper scrotum) or can be ectopic (perineum, contralateral scrotum, or femoral region). UDTs are palpable approximately $70 \%$ of the time [9]; for those that are non-palpable, $55 \%$ are intrabdominal, $30 \%$ are inguinoscrotal, and $15 \%$ are absent or vanishing $[10,11]$. Whether or not the testicle is palpable will influence the surgical approach. 
The management of a UDT should not include imaging. Ultrasound, a commonly used study, is often non-contributory. A meta-analysis of 12 studies showed that ultrasound has both a low sensitivity (45\%) and specificity (78 \%) in locating a non-palpable testis. Using a pretest probability of around $55 \%$ that a non-palpable testis was in fact intraabdominal, the probability of a non-

palpable testis being intraabdominal following a positive and negative ultrasound was 64 and $49 \%$, respectively. In other words, $49 \%$ of boys with a nonpalpable testis who have a negative ultrasound will in fact have an intraabdominal testis [12]. Basing management decisions on an ultrasound can have significant negative consequences. For instance, if an evaluation for UDT is mistakenly deemed complete after a negative ultrasound, a substantial number of boys will harbor a cryptorchid testis and be exposed to the risks that are associated with an intraabdominal location. Consequently, ultrasound should not be performed for identification of a UDT, even in obese or uncooperative boys where physical exam can be difficult.

Other modalities, such as CT and MRI, are not recommended due to cost, the need for anesthesia, availability, and in the case of $\mathrm{CT}$, ionizing radiation. A recent meta-analysis identified eight studies evaluating 193 non-palpable testes and found that the overall sensitivity for detection using MRI was $62 \%$. The sensitivity of MRI decreased to $55 \%$ if the testis was located intraabdominally, but increased to $86 \%$ if it was inguinoscrotal. MRI was a poor test for identifying atrophied testes in this analysis [13]. In sum, no present imaging modality can determine with absolute certainty that a testicle is absent. Therefore, diagnostic imaging should not be routinely obtained in the evaluation of a boy with cryptorchidism, as it can obscure the diagnosis of cryptorchidism.

Surgical exploration, often using minimally invasive laparoscopy, is the gold standard for the diagnosis of a non-palpable testis. If the testicle is visualized, an orchiopexy (also referred to as orchidopexy), which is the surgical relocation of the testis into the scrotum, can be performed. If the spermatic vessels are blind ending, the diagnosis of an absent or vanishing testis is made. In this way, both the diagnosis and treatment can be performed is a single setting.

It is important for providers to discern retractile testes from UDTs. Retractile testes are best diagnosed by manually grasping the extrascrotal testis and bringing it down into the scrotum and holding it there for at least $30 \mathrm{~s}$. This maneuver allows the cremasteric muscle to fatigue and helps to discern a retractile testis from an UDT; a retractile testis will remain intrascrotal until the cremasteric reflex is stimulated while a UDT will not. Retractile testes do not necessitate surgical correction with orchiopexy, as they often fully descend by puberty and there is no increased risk of infertility [14]. However, testicular ascent may be higher in these boys compared to boys with testicles always located in the dependent scrotum. The incidence of testicular ascent with a retractile testis ranges widely from 2 to $45 \%$ [15]. This predisposition could be due to an overactive cremasteric reflex, entrapping adhesions, or a foreshortened processus vaginalis. Ascended testes may also be at a higher risk of decreased 


\section{Treatment}

fertility as well as germ cell disorders. Therefore, boys with a retractile testis are recommended to undergo a testicular examination at least annually.

The rationale for the treatment of cryptorchidism is to reduce the risk of its longterm sequelae, including progressive subfertility/infertility, testicular neoplasms, torsion, and cosmetic concerns.

\section{Fertility}

Testicular malignancy

Spermatogenic function has long been observed to be diminished in the setting of a UDT, which can result in subfertility or infertility. There is a small difference in the temperature between the abdomen and the scrotum $\left(2-3{ }^{\circ} \mathrm{C}\right)$, but this warmer environment impairs normal spermatogenesis. These undescended testes have had an arrest in development of the germinal epithelium, with a reduction in the total number of germ cells starting in the first year of life. Fibrotic changes in the stromal connective tissue have also been noted [16]. These histologic changes may play a role in the impaired fertility seen in unilateral, and more significantly, bilateral cryptorchidism. The overall probability of infertility among males with a history of unilateral cryptorchidism is $10 \%$, which is similar to those without a history of cryptorchidism [17•]. Neither the size nor the position of the testicle was related to paternity rates in the unilateral cryptorchid group. However, $50-90 \%$ of patients with bilaterally untreated cryptorchid testes have infertility [18]. This makes cryptorchidism the leading cause of azoospermia [19, 20].

Germ cell transformation is an important risk factor associated with future fertility potential, especially in patients with a normal number of germ cells at the time of orchiopexy. Transformation of germ cells into Ad (dark) spermatogonia, which are the stem cell progenitors for spermatogenesis, occurs around 3-6 months of post-gestational age when a surge of testosterone and gonadotropins occurs. Hadziselimovic et al. showed that if this maturation does not occur, the risk of future infertility is around $90 \%$. These authors argue that boys with cryptorchidism requiring surgery should undergo testicular biopsy at the time of the orchiopexy to determine the quantity of Ad spermatogonia. This would help identify those who might benefit from LHRH analogues [21]. These hormones may help stimulate the replication and maturation of germ cells to increase the chance of future fertility [22].

Cryptorchidism is one of the few clearly established risk factors for testicular malignancy. The pathogenesis may be attributed to undifferentiated gonocytes which remain in the cryptorchid testis postnatally. These can later develop into carcinoma in situ (CIS), and eventually, a germ cell tumor (GCT) [23]. Wood and Elder performed an extensive review of the literature and showed that cryptorchidism increases the relative risk of testicular cancer by 2.75 to 8 , despite earlier reports suggesting that the number was much higher [24]. In a study of almost 1700 Swedish boys who underwent an orchiopexy, Pettersson et al. showed that when performed between the ages of 0-6, 7-9 and 10-12 
years, the relative risk of malignancy was $2.02,2.35$, and 2.27, respectively, compared to age-matched controls without cryptorchidism. On the other hand, the relative risk of malignancy in boys after orchiopexy performed between 13 to 15 and 16 to 19 years was 5.06 and 6.24 , respectively. This suggests that the risk is not only increased after puberty, but it may rise with increasing age.

Fortunately, post-pubertal orchiopexy has become far less common today. The proportion of boys in the Swedish study who were 13 or older at orchiopexy was $27 \%$ in the 1960 s, but decreased to $5 \%$ by the late 1980 s [25••]. Wood and Elder also reported that a properly descended contralateral testis carries no higher risk of testicular cancer. Their meta-analysis also revealed that malignancies of testes that remain cryptorchid are more likely to be seminomas (74\%), while corrected cryptorchid testes are more prone to develop nonseminomatous GCTs (63\%) [24].

Orchiopexy

Orchiopexy has been the mainstay of treatment for cryptorchidism in the USA. In the setting of a palpable testis, the location can often determine which surgical approach will be used. An examination under anesthesia offers an opportunity to better assess, or confirm, the position of the testicle. If it is found in the inguinal region, a transverse inguinal incision is used to divide the gubernacular attachment, separate the cremasterics off of the spermatic cord, isolate and ligate the hernia sac, and mobilize the testis into the scrotum within a subcutaneous or subdartos pouch. If the testis is palpable near the scrotum, or it can easily be brought down to a dependent scrotal position, a scrotal approach can be used. This employs a periscrotal incision to perform the aforementioned steps, with occasionally an inguinal incision also being used if more proximal dissection is needed for mobilization than a scrotal incision allows.

Other techniques can be used if the testis is not able to initially gain the length needed to provide tension-free placement in the scrotum. One such option is a Fowler-Stephens orchiopexy, where the testicular artery is divided. The testis then relies on collateral blood flow from the deferential and external spermatic arteries and can be brought down into the scrotum in either a 1 or 2staged procedure. Additionally, the Prentiss maneuver can be performed, where the transversalis fascia is opened and the testis is passed medial to the inferior epigastric vessels to allow for a more direct path to the scrotum. Alternatively, microvascular autotransplantation to the inferior epigastrics can be used in some cases. Testes that are non-palpable are most commonly explored using a diagnostic laparoscopy; however, abdominal incisions and extended inguinal incisions are utilized as well.

Primary hormonal therapy is another treatment modality that has been used to induce testicular descent, although this has been more widely practiced outside of the USA. Human chorionic gonadotropin (hCG) and gonadotropin releasing hormone $(\mathrm{GnRH})$ have been used both individually and together for this purpose, with the rationale being that endocrine factors, and specifically disorders of the hypothalamic-pituitary-gonadal axis, likely play a role in cryptorchidism. hCG acts to stimulate androgen production by Leydig cells, which mimics the effects of pituitary luteinizing hormone (LH). Similarly, GnRH 
triggers the release of pituitary gonadotropins LH and follicle-stimulating hormone (FSH), causing a temporary increase in gonadal steroidogenesis.

Success rates from hormonal therapy are extremely variable. Reports from prior studies demonstrated an 8-60\% anatomical response, which is considered testicular descent into the scrotum without atrophy [26]. This variation in response is likely due to the different proportions of retractile testes included in these previous studies. Nevertheless, several meta-analysis of the randomized studies show success rates actually around $20 \%$, which after follow-up, decreases to $15 \%$ as a result of secondary re-ascent of the testis [27-29]. Hormonal therapy does seem to have higher rates of success in testicles situated in a distal position compared to a more proximal location. In contrast though, primary orchiopexy has an overall success rate of about $95 \%$. Finally, one study showed that the use of hCG in cryptorchidism was associated with increased apoptosis of spermatogonia compared to cryptorchid testes not treated with hCG. When these boys were followed into adulthood, those that received hCG had a $50 \%$ smaller testicular volume [29]. Thus, hCG treatment may in fact negatively impact spermatogenesis, yet this has not been thoroughly studied and the potential for selection bias exists. For these reasons, the recent AUA guidelines as well as the Nordic consensus panel recommend against the use of hormonal therapy to facilitate testicular descent $[30 \bullet \bullet, 31]$.

Timing of referral

The timing for initial referral and subsequent surgical correction has been debated. At question is whether or not there is irreversible and progressive damage to the testis if left cryptorchid, and if full recovery is possible, does the length of time that the testis was undescended matter?

At birth, the prevalence of cryptorchidism is around $3 \%$, but the prevalence declines to about $1 \%$ by 3 months (corrected for gestational age) and remains around $1 \%$ at 1 year [4]. Hence, the majority of cases of congenital cryptorchidism resolve spontaneously during the "mini-puberty" in early infancy that results in a surge of endogenous testosterone. A retrospective study of 1235 boys with cryptorchidism revealed that all patients with eventual spontaneous descent initially presented before 6 months (corrected for gestational age). Of those boys initially presenting beyond 6 months of age, none had spontaneous testicular descent [32]. Therefore, testicles that do not descend by 6 months are unlikely to do so spontaneously.

Recommendations for the age at which orchiopexy should be performed have decreased over time. In 1986, the American Academy of Pediatrics suggested surgery between the ages of 4 and 6 years. This recommendation was lowered to 1 year of age a decade later. The earlier timeframe was advised after recognizing that the amount of germ cells and tubular growth in cryptorchid testes was decreased after 2 years of age [33]. Kollin et al. demonstrated a beneficial effect of early surgery on testicular growth. They randomized boys to surgery at 9 months or 3 years of age, and then assessed testicular volume with interval ultrasounds compared to the contralateral descended testis up until age 4. Orchiopexy at 9 months was associated with an increased testicular volume of the relocated testicle at 2,3, and 4 years compared to the initial volume at 6 months. On the other hand, there was no significant growth seen in the group treated at 3 years of age. This suggests a partial "catch-up growth" with early 
intervention, at least up until age 4 [34]. Therefore, referral to a surgical specialist for congenital cryptorchidism should occur at 6 months (corrected for gestational age) in order to facilitate correction within the next year. Acquired cryptorchidism discovered later in life should be referred soon after the diagnosis is made $[30 \bullet \bullet$.

Bilateral non-palpable testes, which occurs in about $20-30 \%$ of patients with cryptorchidism, requires additional workup for disorders of sexual development (DSD) [4]. Infants who appear to be phenotypically male but have nonpalpable gonads should be evaluated for congenital adrenal hyperplasia (CAH). In these instances, circumcision should not be performed, as patients that are genetically female ( $46 \mathrm{XX}$ ) with severe $\mathrm{CAH}$ can be misidentified as a male with bilateral cryptorchidism. CAH is essential to diagnose early, as these infants can develop severe electrolyte disturbances (hyponatremia and hyperkalemia) as well as shock. In the setting of phallic abnormalities (hypospadias or micropenis) with either unilateral or bilateral cryptorchidism, DSD should be considered. As a result, an infant with bilateral non-palpable testes should warrant monitoring of electrolytes, karyotype analysis, and a hormonal profile (including 17-hydroxyprogesterone levels, LH, FSH, testosterone, and androstenedione), with referral to a pediatric endocrinologist and pediatric urologist $[30 \bullet \bullet]$.

Patients with bilateral cryptorchidism who do not have CAH should be evaluated to see if the testicles are present. Differentiation of the male genital tract and external genitalia is dependent on testosterone and müllerian inhibiting substance (MIS). If the patient has a normal male phenotype, two possibilities exist: either both testes are intraabdominal or they have vanished in utero (bilateral congenital anorchia). The former is about 20 times more common than bilateral vanishing testes syndrome [35]. MIS is produced by the Sertoli cells of the testis and can assess whether viable testicular tissue is present. Other hormonal tests, such as inhibin $\mathrm{B}, \mathrm{LH}, \mathrm{FSH}$, and testosterone can also be obtained. Sertoli cells produce MIS and inhibin B in response to FSH, while Leydig cells produce testosterone when stimulated by either endogenous LH or exogenous hCG.

In the setting of bilateral non-palpable testes and a $46 \mathrm{XY}$ karyotype, undetectable plasma concentrations of MIS and inhibin B as well as elevated plasma FSH is adequate to make the diagnosis of anorchia. An hCG stimulation test has also been used in the past, where serum testosterone is measured before and after doses of hCG are administered. A low testosterone response suggests an absence of testicular tissue. However, Misra et al. reported that the positive predictive value (PPV) of low testosterone response to hCG for anorchia was only $57 \%$, while the PPV for unmeasurable MIS concentration was $92 \%$ [36]. Therefore, the hCG stimulation test can generally be avoided, and if MIS is present, the patient should proceed to surgery to preserve testicular function.

\section{Conclusion}

Cryptorchidism is a relatively common finding in boys which should be actively screened for in both the newborn and pediatric populations. Prematurity 
remains the single greatest risk factor, although maternal and genetic factors likely contribute as well. The diagnosis is often made by physical exam alone and should be determined early in order to facilitate timely correction.

Orchiopexy remains the treatment of choice with a success rate significantly higher than hormonal therapy, which is no longer recommended. Early treatment can reduce, but not eliminate, the risk of decreased fertility and testicular malignancy.

\section{Compliance with Ethical Guidelines}

\section{Conflict of Interest}

David Kurz declares that he has no competing interest. Gregory Tasian declares that he has no competing interest.

Human and Animal Rights and Informed Consent

This article does not contain any studies with human or animal subjects performed by any of the authors.

\section{References and Recommended Reading}

Papers of particular interest, published recently, have been highlighted as:

- Of importance

$\bullet \quad$ Of major importance

1. Sijstermans K, Hack WW, Meijer RW, et al. The frequency of undescended testis from birth to adulthood: a review. Int J Androl. 2008;31:1.

2. Sampaio FJ, Favorito LA. Analysis of testicular migration during the fetal period in humans. J Urol. 1998;159:540.

3. Scorer CG. The descent of the testis. Arch Dis Child. 1964;39:605.

4. Berkowitz GS, Lapinski RH, Dolgin SE, et al. Prevalence and natural history of cryptorchidism. Pediatrics. 1993;92:44.

5. Jensen MS, Toft G, Thulstrup AM, et al. Cryptorchidism concordance in monozygotic and dizygotic twin brothers, full brothers, and half-brothers. Fertil Steril. 2010;93:124.

6. Hackshaw A, Rodeck C, Boniface S. Maternal smoking in pregnancy and birth defects: a systematic review based on 173687 malformed cases and 11.7 million controls. Hum Reprod Update. 2011;17:589.

7. Snijder CA, Kortenkamp A, Steegers EAP, et al. Intrauterine exposure to mild analgesics during pregnancy and the occurrence of cryptorchidism and hypospadia in the offspring: the generation R study. Hum Reprod. 2012;27:1191-201.

8. Martin O, Shialis T, Lester J. Testicular dysgenesis syndrome and the estrogen hypothesis: a quantitative meta-analysis. Cien Saude Colet. 2008;13:1601.

9. Smolko MJ, Kaplan GW, Brock WA. Location and fate of the nonpalpable testis in children. J Urol. 1983;129:1204.

10. Merguerian PA, Mevorach RA, Shortliffe LD, et al. Laparoscopy for the evaluation and management of the nonpalpable testicle. Urology. 1998;51:3.

11. Denes FT, Saito FJ, Silva FA, et al. Laparoscopic diagnosis and treatment of nonpalpable testis. Int Braz J Urol. 2008;34:329.

12. Tasian GE, Copp HL. Diagnostic performance of ultrasound in nonpalpable cryptorchidism: a systematic review and meta-analysis. Pediatrics. 2011;127:119.

13. Krishnaswami S, Fonnesbeck C, Penson D, et al. Magnetic resonance imaging for locating nonpalpable undescended testicles: a meta-analysis. Pediatrics. 2013;131:6.

14. Shapiro E. The risk of retractile testes becoming ascending testes. Rev Urol. 2006;8(4):231-2.

15. Stec AA, Thomas JC, DeMarco RT, et al. Incidence of testicular ascent in boys with retractile testes. J Urol. 2007; 178:1722.

16. Mancini RE, Rosemberg E, Cullen M, et al. Cryptorchid and scrotal human testes. I. Cytological, cytochemical and quantitative studies. J Clin Endocrinol Metab. $1965 ; 25: 927$.

17. Miller KD, Coughlin MT, Lee PA. Fertility after unilateral cryptorchidism. Paternity, time to conception, pretreatment testicular location and size, hormone and sperm parameters. Horm Res. 2001;55(5):249-53.

This study addresses the important and freqently discussed impact cryptorchidism has on fertility. 
18. Lee PA, Coughlin MT. Fertility after bilateral cryptorchidism. Evaluation by paternity, hormone, and semen data. Horm Res. 2001;55(1):28-32.

19. Hadziselimovic F, Herzog B. The importance of both an early orchidopexy and germ cell maturation for fertility. Lancet. 2001;358(9288):1156-7.

20. Hadziselimovic F, Herzog B. Importance of early postnatal germ cell maturation for fertility of cryptorchid males. Horm Res. 2001;55:6-10.

21. Hadziselimovic F, Zivkovic D, Bica DTG, et al. The importance of mini-puberty for fertility in cryptochidism. J Urol. 2005; 174:1536-9.

22. Chung E, Brock GB. Cryptorchidism and its impact on male fertility: a state of art review of current literature. Can Urol Assoc J. 2011;5(3):210-4.

23. Sonne SB, Almstrup K, Dalgaard M, et al. Analysis of gene expression profiles of microdissected cell populations indicates that testicular carcinoma in situ is an arrested gonocyte. Cancer Res. 2009;69:5241.

24. Wood HM, Elder JS. Cryptorchidism and testicular cancer: separating fact from fiction. J Urol. 2009;181(2):452-61.

25.• Pettersson A, Richiardi L, Nordenskjold A, et al. Age at surgery for undescended testis and risk of testicular cancer. N Engl J Med. 2007;356(18):1835-41.

This population-based cohort study demonstrates the association between cryptorchidism and testicular malignancy and how timing of orchiopexy may modify the risk.

26. Thorsson AV, Christiansen P, Ritzén M. Efficacy and safety of hormonal treatment of cryptorchidism: current state of the art. Acta Paediatrica. 2007;96:628-30.

27. Pyorala S, Huttunen NP, Uhari M. A review and metaanalysis of hormonal treatment of cryptorchidism. J Clin Endocrinol Metab. 1995;80:2795-9.
28. Henna MR, Del Nero RG, Sampaio CZ, et al. Hormonal cryptorchidism therapy: systematic review with metanalysis of randomized clinical trials. Pediatr Surg Int. 2004;20:357-9.

29. Ong C, Hasthorpe S, Hutson JM. Germ cell development in the descended and cryptorchid testis and the effects of hormonal manipulation. Pediatr Surg Int. 2005;21:240-54.

$30 . \bullet$ Kolon TF, Herndon CDA, Baker LA, et al. Evaluation and treatment of cryptorchidism: AUA guideline. J Urol. 2014;192(2):337-45.

This guideline offers a detailed summary of the natural history and risk factors for cryptorchidism, as well as the current AUA recommendations for both its diagnosis and managemen.

31. Ritzén EM, Bergh A, Bjerknes R, et al. Nordic consensus on treatment of undescended testes. Acta Paediatr. 2007;96:638-43.

32. Wenzler DL, Bloom DA, Park JM. What is the rate of spontaneous testicular descent in infants with cryptorchidism? J Urol. 2004;171(2 Pt 1):849-51.

33. Mengel W, Hienz A, Sippel WG, et al. Studies on cryptorchidism: a comparison of histological findings in the germinative epithelium before and after the second year of life. J Pediatr Surg. 1974;9:445-50.

34. Kollin C, Karpe B, Hesser U, et al. Surgical treatment of unilaterally undescended testes: testicular growth after randomization to orchiopexy at age 9 months or 3 years. J Urol. 2007;178:1589-93.

35. Boisen KA, Kaleva M, Main KM, et al. Difference in prevalence of congenital cryptorchidism in infants between two Nordic countries. Lancet. 2004;363:1264.

36. Misra M, MacLaughlin DT, Donahoe PK, et al. Measurement of Mullerian inhibiting substance facilitates management of boys with microphallus and cryptorchidism. J Clin Endocrinol Metab. 2002;87:3598-602. 